\title{
Capsule Commentary on Peltz-Sinvani et al., Low ALT Levels Independently Associated with 22-Year All-Cause Mortality among Coronary Heart Disease Patients
}

\author{
Kirk V. Shepard II, MD \\ Department of Internal Medicine, Medical College of Wisconsin, Milwaukee, WI, USA.
}

J Gen Intern Med 31(2):226

DOI: $10.1007 / \mathrm{s} 11606-015-3544-7$

(c) Society of General Internal Medicine 2015

$\mathrm{L}$ ow ALT has been associated with frailty and mortality in other populations. In this study, Peltz-Sinvani et al. ${ }^{1}$ assessed the association between low ALT $(<17$ IU/L) blood levels and long-term (22.8 years) all-cause mortality in patients with stable coronary heart disease. The authors used the Bezafibrate Infarction Prevention (BIP) registry population, which contains over 15,000 patients 40 to 74 years of age with stable coronary heart disease who have been followed continuously for over two decades. ${ }^{2}$ In their analysis they excluded patients with elevated ALTs.

The authors found that the cumulative probability of allcause mortality was significantly higher in patients with low ALT than in patients with higher ALT levels (65.6\% vs. $58.4 \%, \log$-rank $p<0.001)$. Furthermore, multivariate analysis adjusted for multiple established predictors of mortality demonstrated that low ALT is independently associated with an $11 \%$ greater long-term mortality risk (HR 1.11 [95\% confidence interval: 1.03-1.19; adjusted $P<0.01]$ ). PeltzSinvani concluded that low ALT levels are associated with increased long-term mortality amongst middle-aged patients with stable coronary heart disease, even after adjusting for other, well-established risk factors for mortality.

The reported associations may have been confounded by several factors. First, the study was conducted as a retrospective analysis and thus could not account for potential bias or confounders from the BIP registry population. Second, while
Peltz-Sinvani et al. do identify the study population as patients without liver disease and with stable coronary heart disease, they do not comment on other comorbidities that patients may have had, which may or not be associated with abnormal ALT levels. Lastly, older age, female sex, lower BMI, and lower height are all independent predictors of low ALT and may possess their own intrinsic factors that affect long-term mortality.

The ability to assess long-term mortality, particularly in an aging population, is important in treatment planning for both early and advanced disease. Peltz-Sinvani et al.'s findings suggest that ALT blood levels may be helpful in risk stratification of patients with stable coronary heart disease. However, additional studies are needed to explain the association, and the appropriate intervention must be elucidated.

Corresponding Author: Kirk V. Shepard, II, MD; Department of Internal MedicineMedical College of Wisconsin, Milwaukee, WI, USA (e-mail: kishepard@mcw.edu).

\section{Compliance with ethical standards:}

Conflicts of interest: The author has no conflicts of interest with the material in this article.

\section{REFERENCES}

1. Peltz-Sinvani N, et al. Low ALT levels independently associated with 22 year All-cause mortality among coronary heart disease patients. J Gen Intern Med. 2015. doi:10.1007/s11606-015-3480-6.

2. Goldbourt $\mathbf{U}$, et al. Rationale and design of a secondary prevention trial of increasing serum high-density lipoprotein cholesterol and reducing triglycerides in patients with clinically manifest atherosclerotic heart disease (the Bezafibrate Infarction Prevention Trial). Am J Cardiol. 1993;71(11):909-15. 